\title{
Design and Component Test of a Tiny Processor Based on the SFQ Technology
}

\author{
N. Yoshikawa, F. Matsuzaki, N. Nakajima, K. Fujiwara, K. Yoda, and K. Kawasaki
}

\begin{abstract}
An eight-bit SFQ processor has been designed and some key components have been tested to confirm feasibility of the large-scale SFQ digital circuit. The designed processor is composed of a one-bit ALU, two eight-bit registers with local clock generators, an instruction register, a five-bit program counter, a state controller, and a 32-byte register file. A bit-serial architecture and a distributed local clock architecture, where each register has its own local clock generator, have been employed in order to increase the local clock frequency. The target clock frequency is $16 \mathrm{GHz}$ and $10 \mathrm{GHz}$ for the NEC $2.5 \mathrm{kA} / \mathrm{cm}^{2}$ and Hypres $1 \mathrm{kA} / \mathrm{cm}^{2} \mathrm{Nb}$ processes. On the circuit design level, we have used a data-driven self-timed architecture and a binary decision diagram, which reduce the timing design difficulty in high frequency operation. The processor, which contains 7,300 Josephson junctions, has been designed by using a cell-based design methodology with the assistance of a top-down CAD environment. We have successfully tested some important circuit blocks, including a one-bit ALU, eight-bit registers, and a demultiplexer for register files.
\end{abstract}

Index Terms-Binary decision diagram, bit-serial architecture, RSFQ, SFQ processor.

\section{INTRODUCTION}

$\mathbf{T}$ HE most attractive and challenging application of the SFQ digital systems [1] is a high-end computing or server application [2]-[5], where both strong points of the SFQ circuits, high speed and low power, are fully utilized. We have been developing tiny SFQ processors to show the feasibility of largescale SFQ circuits. We call this project Tiny Processor Project in Yokohama (TIPPY). The aim of the project is to realize the simplest SFQ processor having complete functionality as a general purpose computing system. For practical demonstration of the processor operation, we are planning to implement all circuit components on a single chip of a $5 \mathrm{~mm}$ die size. Throughout the project, we also expect to encounter unforeseen problems when designing the large-scale circuits, and to find appropriate architectures for the SFQ digital system. Design methodologies and $\mathrm{CAD}$ tools are also being developed to design the large-scale system.

The first version of our SFQ processor, TIPPY1, is an 8-bit processor containing an 8-bit ALU and an accumulator (ACC). It is composed of 7,800 Josephson junctions (JJ) and occupies the area $10,500 \mu \mathrm{m} \times 7,500 \mu \mathrm{m}[6]$. Its clock frequency is very slow $(670 \mathrm{MHz})$ for a $1 \mathrm{kA} / \mathrm{cm}^{2} \mathrm{Nb}$ process, because the ALU is

Manuscript received August 6, 2002. This work was supported by the Ministry of Education, Culture, Sports, Science and Technology under the SFQ project.

The authors are with the Department of Electrical and Computer Engineering, Yokohama National University, Yokohama, Japan (e-mail yoshi@yoshilab.dnj.ynu.ac.jp).

Digital Object Identifier 10.1109/TASC.2003.813892

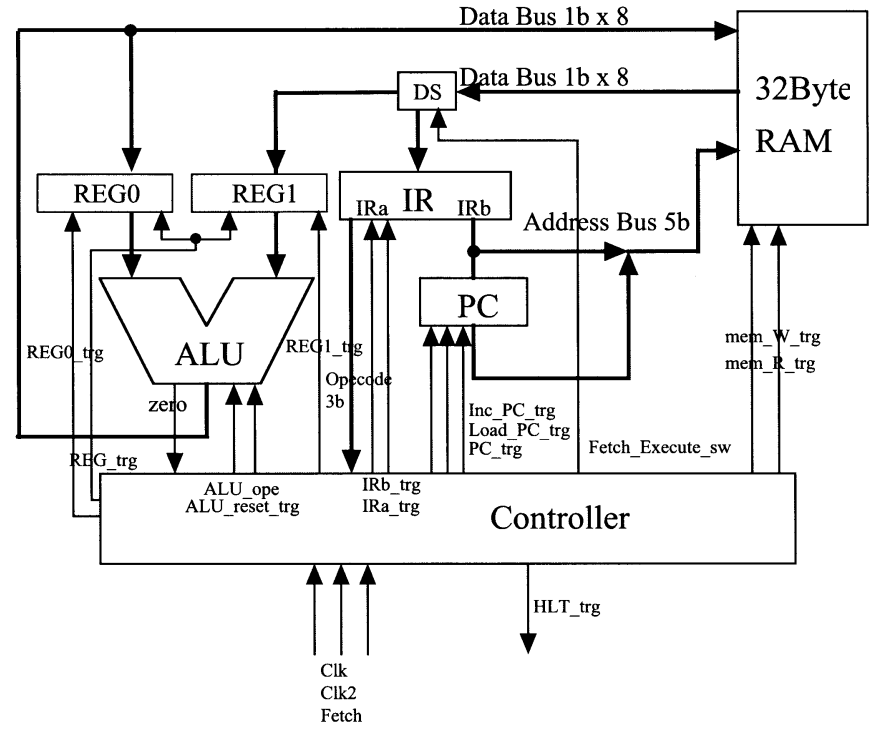

Fig. 1. A block diagram of the 8-bit SFQ microprocessor, TIPPY3.

composed of a simple ripple carry adder that limits the critical speed of the processor. However, it also turned out that a CMOS processor having the same architecture is ten times slower than TIPPY1 assuming a $0.6 \mu \mathrm{m}$ CMOS process.

The second version of our SFQ processor, TIPPY2, employed a bit-serial architecture to increase the local clock frequency and decrease the circuit size [7]. As a result, it operates at $6.25 \mathrm{GHz}$, contains 5,118 JJ and occupies the area 7,300 $\mu \mathrm{m} \times 7,000 \mu \mathrm{m}$, for the $1 \mathrm{kA} / \mathrm{cm}^{2} \mathrm{Nb}$ process.

The third version, TIPPY3, has been designed for the purpose of real implementation and demonstration of the SFQ processor by modifying the design of TIPPY2. Their main difference is that the ACC in TIPPY2 is replaced with two registers in TIPPY3, which are placed adjacent to the ALU to maintain precise timing of the ALU operation at high speed. In the TIPPY3 design, a floor plan is carefully considered to decrease the routing path length between circuit blocks. As a result, all passive transmission lines in TIPPY2 are removed in TIPYY3.

In this paper we will describe the design details of TIPPY 3 and show their functional simulation results using a logic simulator. Component test results of some main circuit blocks will also be shown.

\section{MiCROPROCESSOR ARCHITECTURE}

A block diagram of the 8-bit SFQ processor, TIPPY3, is shown in Fig. 1. The processor is composed of a one-bit 


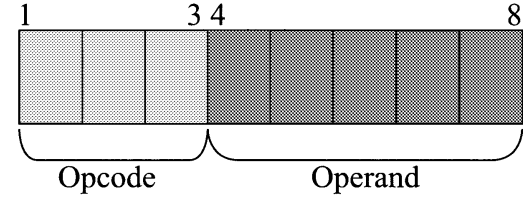

Fig. 2. Instruction format of TIPPY3.

TABLE I

INSTRUCTION SET OF TIPPY3

\begin{tabular}{lll}
\hline $\begin{array}{l}\text { Instruction } \\
\text { Example }\end{array}$ & $\begin{array}{l}\text { Instruction } \\
\text { Name }\end{array}$ & Meaning \\
\hline HLT & Halt & Stop \\
ADD & Add & REG0 + REG1 $\rightarrow$ REG0 \\
LD R1 & Load & Mem[R1] $\rightarrow$ REG1 \\
ST R2 & Store & REG0 $\rightarrow$ Mem[R2] \\
SKZ & Skip if Zero & If(REG0=0)PC+1 $\rightarrow$ PC \\
JMP R3 & Jump & R3 $\rightarrow$ PC \\
MOVE & Move & REG1 $\rightarrow$ REG0 \\
\hline
\end{tabular}

ALU, two eight-bit registers (REG0 and REG1), an instruction register (IR), a five-bit program counter (PC), a data selector (DS), a state controller, and a 32-byte register file (RAM). Its basic structure is similar to TIPPY2 except that TIPPY3 has two registers adjacent to the ALU. This revision guarantees the precise timing of the ALU operation at high clock frequency. The data bus width is 1-bit, therefore all data in the data path are processed serially. This bit-serial architecture increases the local clock frequency up to the limit of the SFQ circuit. Though the system cycle frequency is reduced in the bit-serial architecture, it decreases the system size. Therefore we can say that the advantage of the speed of the SFQ circuits is utilized to reduce the system complexity [5].

Fig. 2 shows the instruction format of TIPPY3. The 8-bit instruction is composed of a 3-bit opcode and a 5-bit operand. The 3-bit opcode determines the processor operations, which consists of seven instructions as listed in Table I. In TIPPY3 a MOVE operation is added to move the data from the REG1 to the REG0. The 5-bit operand directly specifies the data address of the 32-byte RAM. It should be noted here that though TIPPY 3 has only seven instructions, it includes a basic function for the conditional branch operation, which is necessary for general purpose computing.

The internal state of TIPPY3 has eight phases: The first four phases are used for the instruction fetch, and the second four phases are the instruction decode, the ALU setup, the execute and the store-result phases. In the instruction fetch phase, the data on the RAM specified by the PC are sent to the IR. In the instruction decode phase, the 3-bit opcode in the IR is sent to the controller. In the ALU setup phase, the ALU function is setup by the controller according to the instruction. After the execute phase, where the ALU operations are executed, the data in REG0 is moved to the RAM in the store-result phase. No pipelining technique is used for the simplicity of the system.

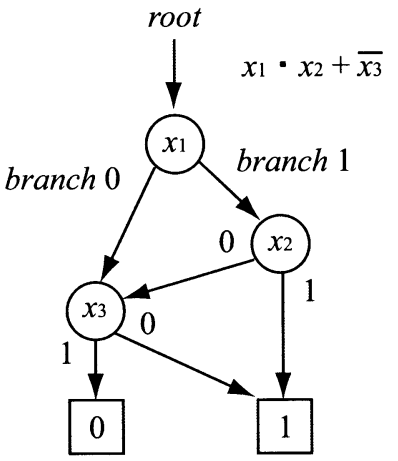

(a)

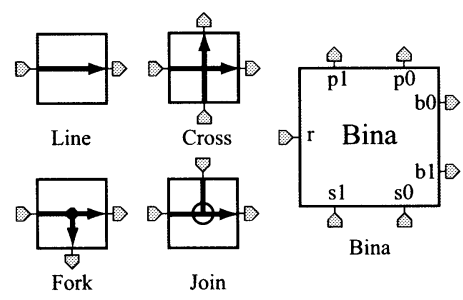

(b)

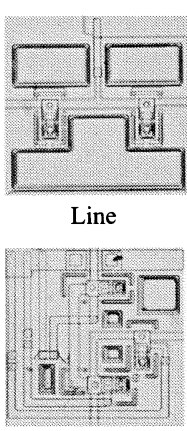

Fork

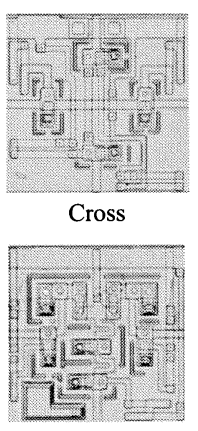

Join

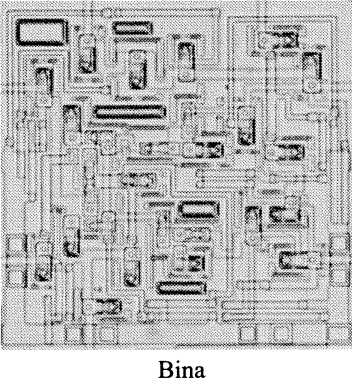

(c)
Fig. 3. (a) A binary decision diagram. The diagram shows the logical function $x 1 x 2+\overline{x 3}$. (b) Symbols and (c) photographs of the basic cells in the BDD SFQ standard cell library.

\section{DeSIGN Methodology}

We have used several design methodologies and techniques when designing the SFQ processor as described in the following subsections.

\section{A. Data-Driven Self-Timed Architecture}

In the data-driven self-timed (DDST) architecture [8], the data communications between circuit blocks are carried out by using dual-rail lines, by which we can eliminate the globally synchronous clock that is necessary in the conventional SFQ circuits.

\section{B. Binary Decision Diagram}

We have used the binary decision diagram (BDD) for logic synthesis and circuit implementation [9]. Fig. 3(a) shows the BDD representing the logical function $f=x 1 x 2+\overline{x 3}$. The BDD is composed of binary switches having one input (root) and two outputs (branch0 and branch1), where an SFQ pulse entering the root is switched into one of two branches according to its internal state. Because the binary switch is implemented by using the D2 flip-flop having dual-rail inputs, the BDD SFQ circuit is one of the DDST logic circuits. The advantage of the BDD SFQ circuit is the simplicity in the timing design and the short delay due to its asynchronous nature. Note that the logic synthesis methodology by using the BDD is well established [9]. 


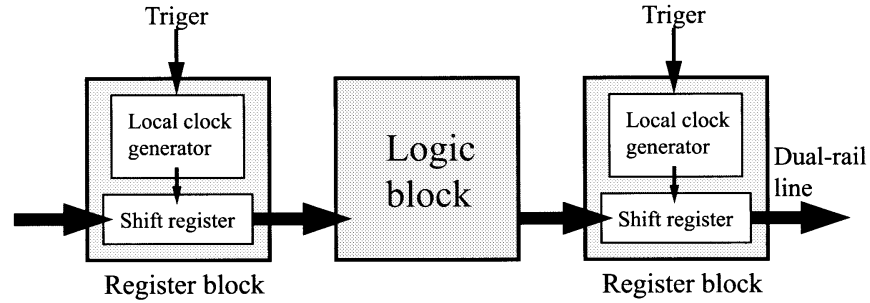

Fig. 4. Conceptual diagram of distributed local clock (DLC) architecture.

\section{Distributed Local Clock Architecture}

Because we employ the bit-serial architecture for the data path, the data communication between circuit blocks is carried out serially at very high clock frequency, above $10 \mathrm{GHz}$. It seems very difficult to distribute clock signals to each circuit block by using a single clock generator. In the distributed local clock (DLC) architecture, each register has its own local clock generator and generates high-speed bit-serial data by itself as illustrated in Fig. 4. The controller only provides an SFQ trigger pulse to activate the read-out operation of the register block.

\section{Cell-Based Design Methodology}

We have used cell-based design methodology to simplify the layout design [10]. In our cell-based design approach, the circuit is designed by simply placing the tile-shaped basic cells. Fig. 3(b) and 3c shows a symbol view and a photograph of the basic cells in our BDD SFQ cell library. In the figure, the Bina works as a binary switch of the BDD, where input node $\mathrm{s} 0, \mathrm{~s} 1$ are used to set the internal state. The $\mathrm{r}, \mathrm{b} 0$, and $\mathrm{b} 1$ correspond to root, branch0 and branch1, respectively. We have implemented the BDD SFQ cell library [11] by using the Hypres $1 \mathrm{kA} / \mathrm{cm}^{2}$ $\mathrm{Nb}$ standard process. We have also added our BDD cells to the CONNECT cell library for the NEC $2.5 \mathrm{kA} / \mathrm{cm}^{2} \mathrm{Nb}$ standard process [12].

\section{E. Logic Level Simulation}

We have developed a logic simulation environment based on a Verilog HDL, by which we can check the functionality and timing violation of the circuits by changing the bias voltage [13]. We can also execute small benchmark test programs by using our logic simulator.

\section{Circuit Design Details}

\section{A. 8-Bit Bit-Serial Register}

As stated above, we use the DLC architecture for the register design. Fig. 5 shows a circuit schematic of the 8-bit bit-serial register, which is composed of an 8-bit ladder type clock generator and an 8-bit DDST shift register. In the write operation of the register, the data stored in the register is rewritten every time the data is input, and during this time no datum is output. In the read operation, when a trigger pulse, (the REG_trg), is applied, the clock generator generates 8 pulses, then the data stored in the register is pushed out. The register design is modified from the previous version so that the propagation delay, the time it takes for the output to appear after the incidence of the trigger pulse,

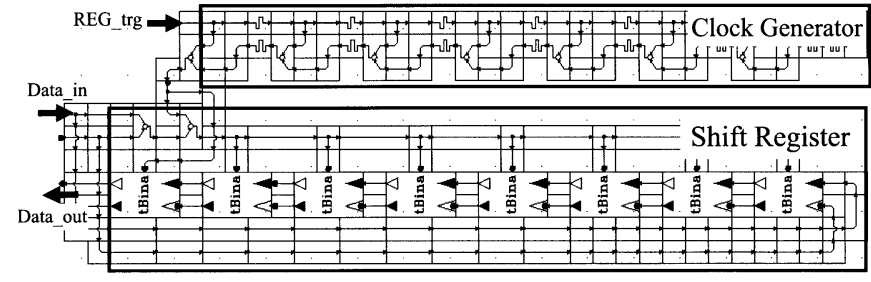

Fig. 5. A circuit schematic of the 8-bit bit-serial register.

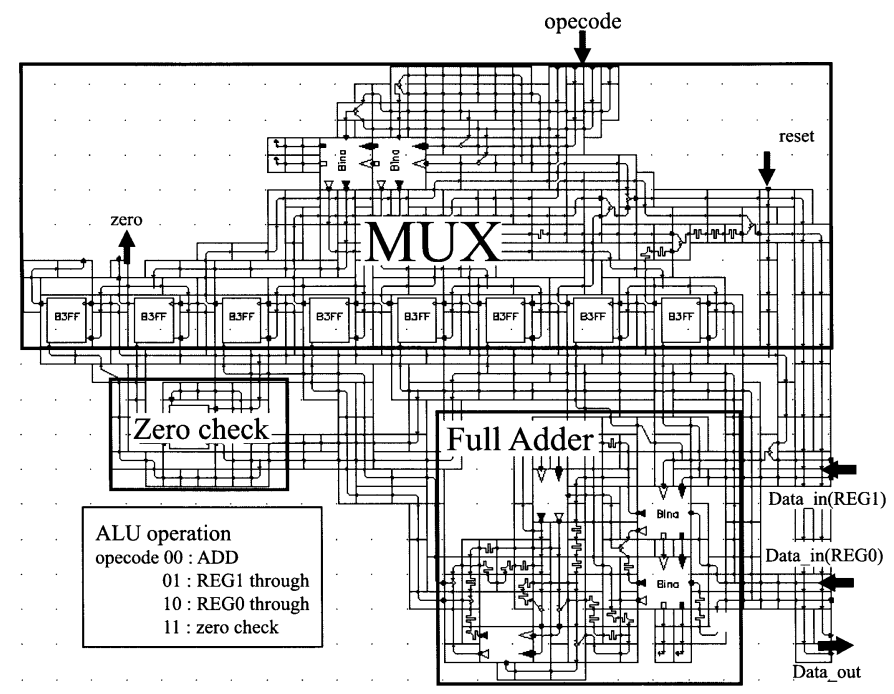

Fig. 6. A circuit schematic of the 1-bit ALU.

is reduced. Circuit simulations by JSIM show that the propagation delay is 144 ps and the DC bias margin is $-26 \% \sim+30 \%$ at $10 \mathrm{GHz}$ assuming the NEC process.

\section{B. $A L U$}

Fig. 6 shows a circuit schematic of the 1-bit ALU, which is composed of a bit-serial full adder, an OR gate to perform the zero check operation, and a multiplexer. The ALU executes four operations: add, zero check, REG0 through and REG1 through. These functionalities are selected by the multiplexer. Logic simulations of the ALU indicate that its DC bias margin is $-30 \%$ $+30 \%$ at $10 \mathrm{GHz}$. Its total junction number is $1710 \mathrm{JJ}$ and the area is $1400 \mu \mathrm{m} \times 1840 \mu \mathrm{m}$.

\section{Controller}

Fig. 7(a) shows a BDD representation of the controller. The controller has seven dual-rail inputs and 11 single-rail outputs. In the figure, the nodes denoted as " 1 ", " 2 " and " 3 " correspond to the state of the opcode, and the nodes "clk", clk2" and "fetch" are the state of the system clocks that determine the internal phase of the processor. The boxes denoted by "PC_trg" mean the output of the PC_trg pulse, for example. It can be seen from the figure that the BDD is useful in representing and implementing the state machine such as state controller of the microprocessor. Fig. 7(b) shows a schematic of the controller. It is found from the logic simulations that its DC bias margin is $-30 \% \sim+30 \%$. Its junction count is $1936 \mathrm{JJ}$ and the area is $1560 \mu \mathrm{m} \times 1440 \mu \mathrm{m}$. 

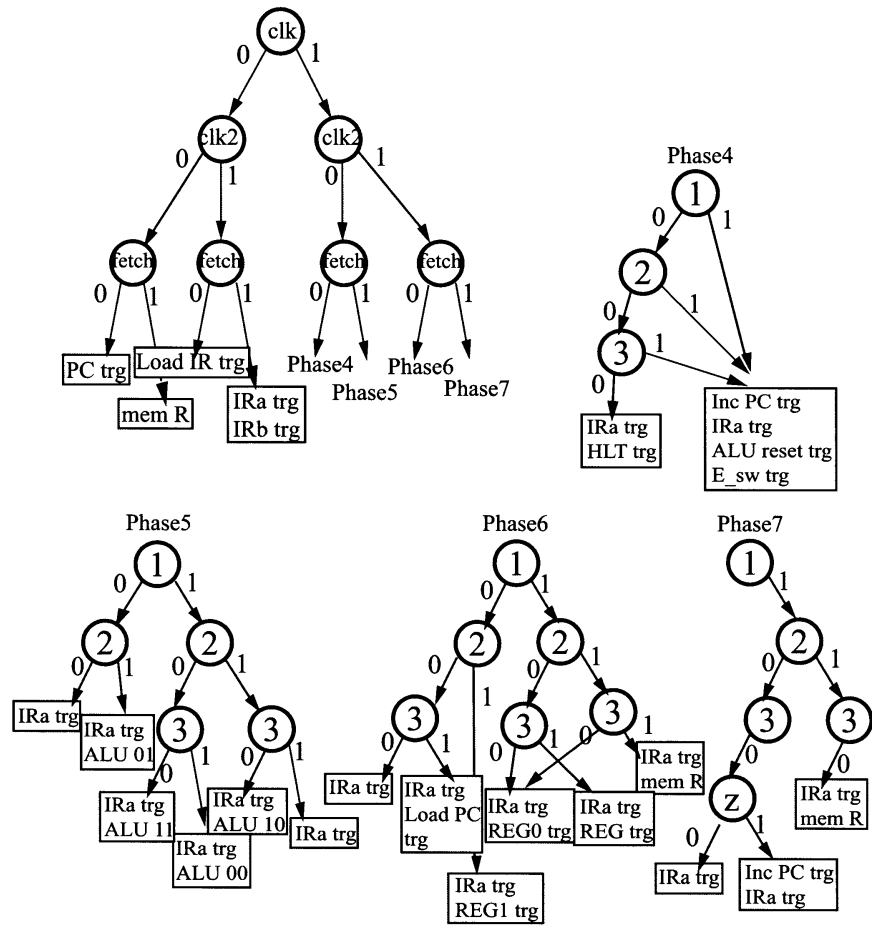

(a)

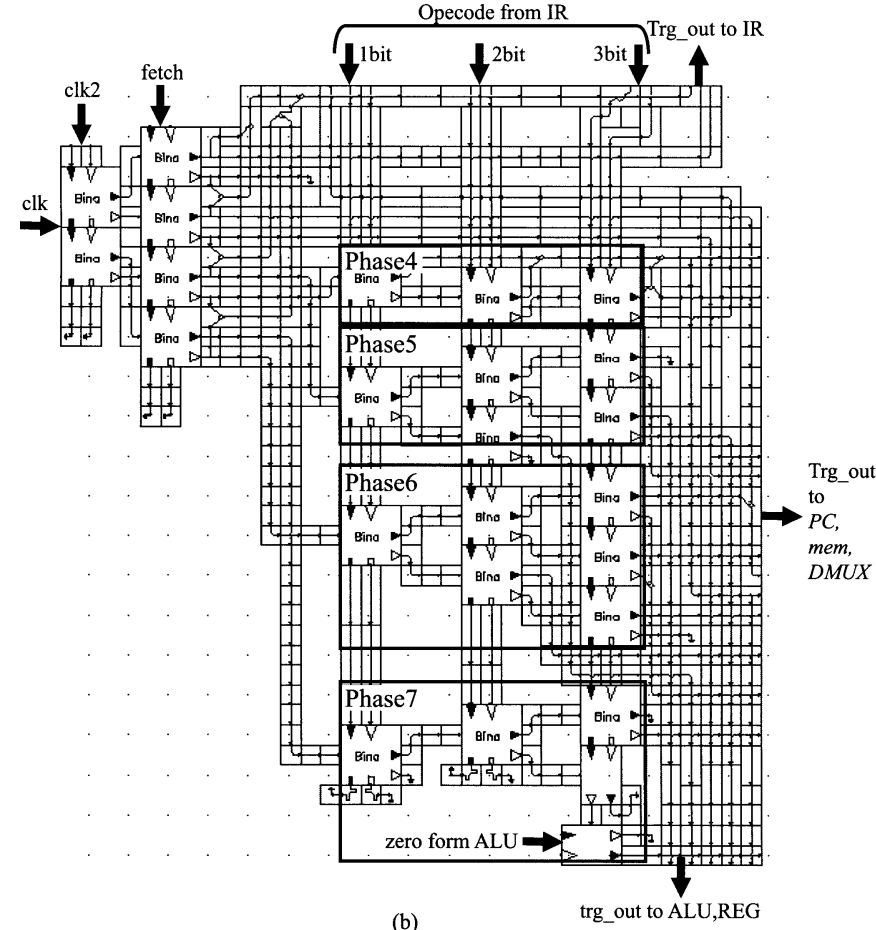

(b)

Fig. 7. (a) The BDD graph and (b) a circuit schematic of the controller.

\section{Microprocessor}

We have designed all other components and integrated them on a single chip except the RAM. Simulated DC bias margins and JJ counts of the components are listed in Table II. Fig. 8 shows a chip layout of TIPPY3, which is implemented by using the NEC process. Chip size is $5 \mathrm{~mm} \times 5 \mathrm{~mm}$ and the total $\mathrm{JJ}$ number is about 7,300. The bias current necessary for the circuit
TABLE II

SimUlated AND Tested DC Bias Margin OF tHE MICROPROCESSOR COMPONENT

\begin{tabular}{lcccc}
\hline \multirow{2}{*}{$\begin{array}{l}\text { Circuit } \\
\text { Component }\end{array}$} & JJ count & \multicolumn{2}{c}{ DC bias margin } & \\
\cline { 3 - 4 } & & $\begin{array}{c}\text { Simulated } \\
@ 10 \mathrm{GHz}\end{array}$ & $\begin{array}{l}\text { Tested } \\
@ 1 \mathrm{KHz}\end{array}$ & \\
\hline ALU & 730 & $-32 \% \sim+32 \%$ & $\pm 3.3 \%$ & Process \\
$\begin{array}{l}\text { Register } \\
\text { (REG) }\end{array}$ & 560 & $-32 \% \sim+36 \%$ & $\begin{array}{l} \pm 6.9 \% \\
(@ 10 \mathrm{GHz})\end{array}$ & Hypres \\
$\begin{array}{l}\text { Data selecter } \\
\text { (DS) }\end{array}$ & 950 & $-26 \% \sim+30 \%$ & -- & NEC \\
$\begin{array}{l}\text { Instruction } \\
\text { Register (IR) }\end{array}$ & 80 & $-30 \% \sim+30 \%$ & $\pm 21.1 \%$ & NEC \\
$\begin{array}{l}\text { Program } \\
\text { Counter (PC) }\end{array}$ & 1090 & $-28 \% \sim+30 \%$ & -- & NEC \\
$\begin{array}{l}\text { Decorder for } \\
\text { RAM }\end{array}$ & 620 & $-30 \% \sim+30 \%$ & $\pm 11.5 \%$ & NEC \\
\hline
\end{tabular}

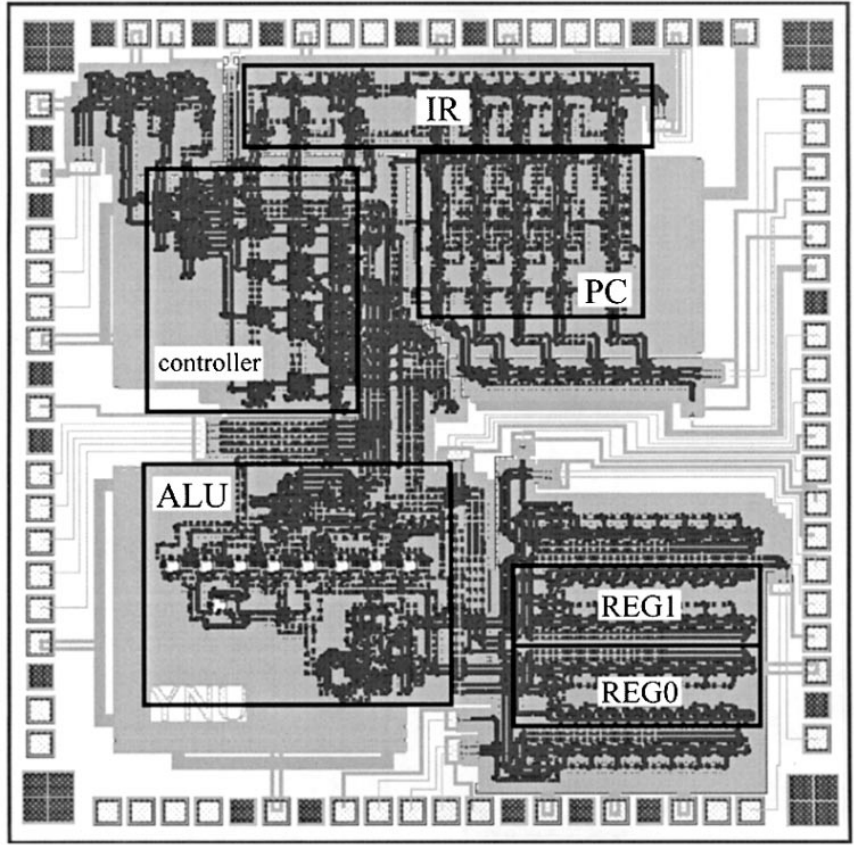

Fig. 8. A chip layout of the 8-bit SFQ microprocessor, TIPPY3, implemented by using the $\mathrm{NEC} \mathrm{Nb} 2.5 \mathrm{kA} / \mathrm{cm}^{2}$ standard process. Chip size is $5 \mathrm{~mm} \times 5 \mathrm{~mm}$.

is $940 \mathrm{~mA}$ in total, therefore total power is $2.35 \mathrm{~mW}$. No passive microstrip line is used in contrast to the previous version.

Fig. 9 shows the logic simulation results of TIPPY3. The figure demonstrates the ADD operation. It is found from the logic simulation that TIPPY3 operates correctly at a local clock frequency of $10 \mathrm{GHz}$ and at a system cycle frequency of $1.2 \mathrm{GHz}$. Fig. 10 shows the test program and its execution results by the logic simulator. We can verify that all the operations are executed correctly from the result.

\section{Component Test Results}

We have implemented some circuit components using the Hypres and NEC Nb standard process and tested them at low 


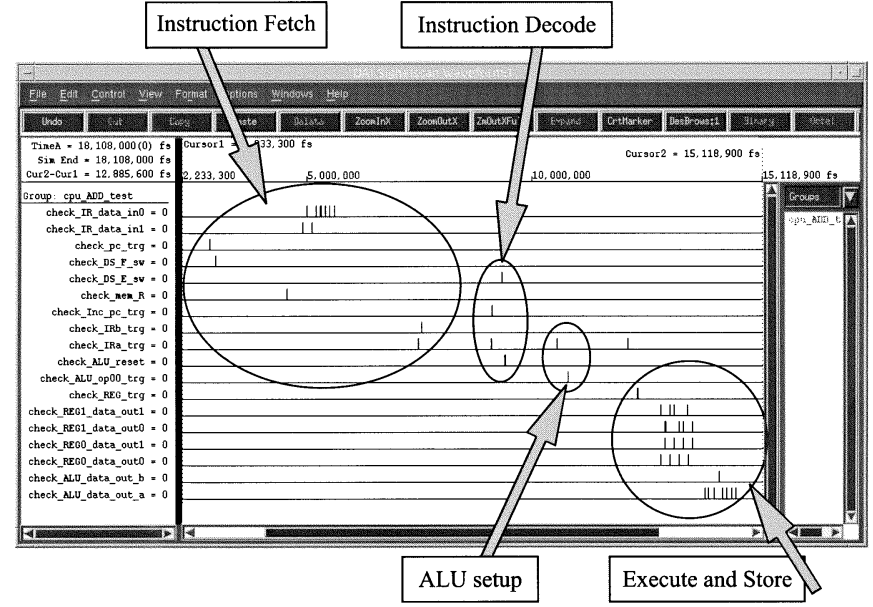

Fig. 9. Logic simulation results of TIPPY3. The figure shows a sequence of the ADD operation. The local clock frequency is $10 \mathrm{GHz}$.

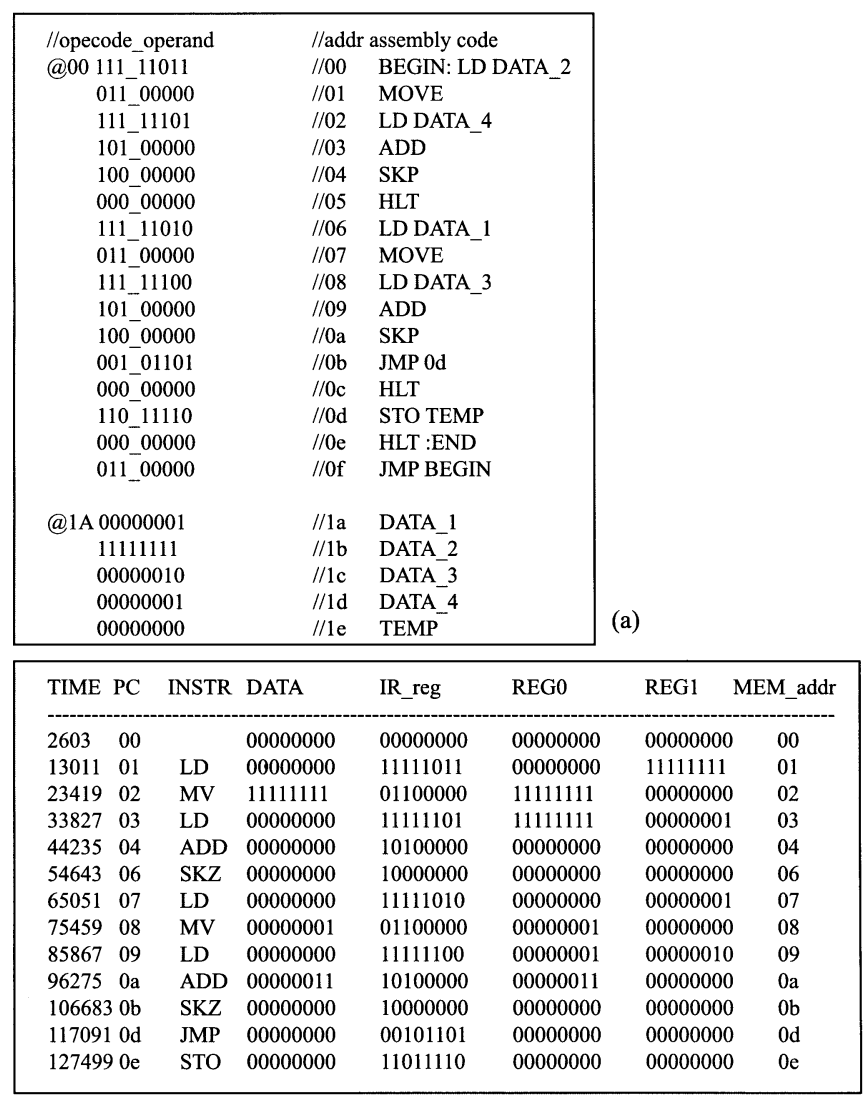

(b)

Fig. 10. (a) A test program and data in the memory. (b) A functional simulation result using the test program. The program tests all operation of the TIPPY3. In the test program, if all operations are executed correctly, the PC and memory address finish at the address " $0 \mathrm{e}$ ".

speed. Tested DC bias margins of the components are listed in Table II. It should be noted that the register has also been tested at $10 \mathrm{GHz}$ using an on-chip high-speed test system [14]. It is found from the table that the tested DC bias margins of large cir- cuits are rather small in contrast to the simulation results. This is probably due to the large bias current flowing along bias lines and ground planes, which induces a magnetic field.

\section{CONCLUSIONS}

We have designed the third version of the tiny SFQ processor, TIPPY3, by using several design approaches such as the DDST, the BDD, the bit-serial architecture, the DLC architecture, and the cell-based design methodology. TIPPY3 contains 7,300 JJ and has been implemented on a single chip of $5 \mathrm{~mm}$ die size. Logic simulations shows that it operates at a local clock frequency of $10 \mathrm{GHz}$ using the NEC $2.5 \mathrm{kA} / \mathrm{cm}^{2}$ standard process. Some key components, including the ALU, the register, the data selector and demultiplexer are tested and their correct operation is confirmed.

\section{ACKNOWLEDGMENT}

The authors would like to thank the CONNECT cell library development teams of Nagoya University, NEC Inc. and CRL for fruitful discussions.

\section{REFERENCES}

[1] K. K. Likharev and V. K. Semenov, "RSFQ logic/memory family: A new Josephson-junction technology for sub-terahertz-clock frequency digital systems," IEEE Trans. Appl. Superconductivity, vol. 1, pp. 1-28, March 1991.

[2] P. Bunyk, A. Y. Kidiyarova-Shevchenko, and P. Litskevitch, "RSFQ microprocessor: New design approaches," IEEE Trans. Appl. Superconductivity, vol. 7, pp. 2697-2704, June 1997.

[3] P. Bunyk and V. K. Semenov, "Design of an RSFQ microprocessor," IEEE Trans. Appl. Superconductivity, vol. 5, pp. 3325-3328, June 1995.

[4] M. Dorojevets, P. Bunyk, D. Zinoviev, and K. K. Likharev, "Superconductor electronic devices for petaflops computing," FED Journal, vol. 10, pp. 3-14, March 1999.

[5] A. Fujimaki, Y. Takai, and N. Yoshikawa, "High-End server based on complexity-reduced architecture for superconductor technology," IEICE Transactions on Electronics, vol. E85-C, no. 3, pp. 612-616, 2002.

[6] F. Matsuzaki, K. Yoda, J. Koshiyama, K. Motoori, and N. Yoshikawa, "Design of small RSFQ microprocessor based on cell-based top-down design methodology," IEICE Trans. Electron., vol. E85-C, pp. 659-664, March 2002.

[7] N. Yoshikawa, F. Matsuzaki, N. Nakajima, and K. Yoda, "Design and component test of a 1-bit RSFQ microprocessor," Physica C, vol. 378-381, pp. 1454-1460, 2002.

[8] Z. J. Deng, N. Yoshikawa, S. R. Whiteley, and T. Van Duzer, "DataDriven self-timed RSFQ digital integrated circuit and system," IEEE Trans. on Applied Superconductivity, vol. 7, pp. 3634-3637, June 1997.

[9] N. Yoshikawa and J. Koshiyama, "Top-Down RSFQ logic design based on a binary decision diagram," IEEE Trans. Appl. Superconductivity, vol. 11, pp. 1098-1101, March 2001.

[10] — "A cell-based design apploach for RSFQ circuits using binary decision diagram," Superconductor Science and Technology, vol. 12, pp. 918-920, 1999.

[11] BDD SFQ Cell library. [Online]. Available: http://www.yoshilab.dnj.ynu.ac.jp/download/download.html

[12] S. Yorozu, Y. Kameda, H. Terai, A. Fujimaki, T. Yamada, and S. Tahara, "A single flux quantum standard logic cell library," Physica $C$, vol. 378-381, pp. 1471-1474, 2002.

[13] N. Yoshikawa, A. Mori, and J. Koshiyama, "Timing design of RSFQ circuits using verilog HDL," Trans. of IEICE, vol. J83-C, pp. 643-650, 2000.

[14] Z. J. Deng, N. Yoshikawa, S. R. Whiteley, and T. V. Duzer, "Data-driven self-timed RSFQ high speed test system," IEEE Trans. Appl. Superconductivity, vol. 7, pp. 3830-3833, December 1997. 